\title{
Neural Network Classification of Tan Spot and Stagonospora Blotch Infection Periods in a Wheat Field Environment
}

\author{
E. D. De Wolf and L. J. Francl
}

Department of Plant Pathology, North Dakota State University, Fargo 58105.

Current address of E. D. De Wolf: Department of Plant Pathology, Ohio State University, Wooster 44691. Accepted for publication 26 October 1999.

\begin{abstract}
De Wolf, E. D., and Francl, L. J. 2000. Neural network classification of tan spot and Stagonospora blotch infection periods in a wheat field environment. Phytopathology 90:108-113.

Tan spot and Stagonospora blotch of hard red spring wheat served as a model system for evaluating disease forecasts by artificial neural networks. Pathogen infection periods on susceptible wheat plants were measured in the field from 1993 to 1998, and incidence data were merged with 24-h summaries of accumulated growing degree days, temperature, relative humidity, precipitation, and leaf wetness duration. The resulting data set of 202 discrete periods was randomly assigned to 10 modeldevelopment or -validation $(n=50)$ data sets. Backpropagation neural networks, general regression neural networks, logistic regression, and parametric and nonparametric methods of discriminant analysis were chosen for comparison. Mean validation classification of tan spot inci-
\end{abstract}

ABSTRACT dence was between $71 \%$ for logistic regression and $76 \%$ for backpropagation models. No significant difference was found between methods of modeling tan spot infection periods. Mean validation prediction accuracy of Stagonospora blotch incidence was 86 and $81 \%$ for backpropagation and logistic regression, respectively. Prediction accuracies of other modeling methods were $\leq 78 \%$ and were significantly different $(P=0.01)$ from backpropagation, but not logistic regression, results. The best backpropagation models of tan spot and Stagonospora blotch incidences correctly classified 82 and $84 \%$ of validation cases, respectively. High classification accuracy and consistently good performance demonstrate the applicability of neural network technology to plant disease forecasting.

Additional keywords: Phaeosphaeria avenaria, Phaeosphaeria nodorum, Pyrenophora tritici-repentis.
Hard red spring wheat (Triticum aestivum L.) and durum wheat (T. turgidum var. durum Desf.) are the predominant crops of the northern Great Plains. Many of the commonly grown cultivars are susceptible to a complex of leaf-spot diseases, including tan spot, caused by Pyrenophora tritici-repentis (Died.) Drechs., and Stagonospora blotch, caused by Phaeosphaeria nodorum (E. Müller) Hedjaroude or Phaeosphaeria avenaria (G. F. Weber) O. Eriksson f. sp. triticea T. Johnson (16). Both diseases occur widely in North America and in wheat-producing regions worldwide, resulting in substantial yield reductions $(15,19,21)$. In North Dakota, severe localized epidemics of both tan spot and Stagonospora blotch occur frequently (16). Severity of these localized epidemics is influenced by local-scale ( 1 to $10 \mathrm{~km}$ ) environmental parameters, including temperature, dew period, relative humidity, and precipitation.

Although host resistance to both diseases is available, widespread adaption of resistance as a management tool has been inhibited by undesirable grain qualities of resistant cultivars. Application of a single, well-timed fungicide effectively manages both tan spot and Stagonospora blotch $(4,23)$. Thus, a disease-forecasting system that accurately predicts these diseases and facilitates management decisions is highly desirable.

Artificial neural networks provide a flexible way to connect disease outcome with environmental and other determinant variables $(3,20)$. Neural networks are similar to regression models in that both develop coefficients that model patterns by evaluation of the relationship between independent and dependent variables.

Corresponding author: E. D. De Wolf; E-mail address: dewolf.4@osu.edu

Publication no. P-1999-1214-01R

(C) 2000 The American Phytopathological Society
However, unlike parametric statistical models, neural networks require no a priori information regarding the appropriate model form (i.e., nonlinear or implicitly linear). In general, neural networks consist of three or more groups of interconnected processing elements that represent sets of equations used by the model. The type of neural network governs how the various processing elements are interconnected and how they process data. Individuals interested in neural networks and other approaches to statistical classification and pattern recognition should refer to texts by Bishop (3) and Ripley (20). Additional information can be found on the Internet at the SAS Institute (Cary, NC) website.

Backpropagation neural networks (BPNN), a common type of neural network, use an iterative presentation of independent variables and observed dependent variables to identify the appropriate form of the model (3). During this iterative process, derivatives of error functions between predicted and observed dependent variables are used to alter model coefficients according to an optimization method such as gradient descent. This iterative process is halted when the error reaches a predetermined criterion or no further improvement is found within a given number of iterations. After which, the functions of the BPNN can be tested by holding model coefficients constant and evaluating the prediction error for validation data (i.e., data not used during model development). Performance of BPNN models can be affected by the number of intermediate processing groups and of processing elements in each group. In addition, computational parameters that control magnitude of weight changes, and random factors such as initialization of weights and order of case presentation, also can influence model performance. These parameters allow for great model flexibility; however, the optimization of numerous parameters often results in an extensive and time-consuming evaluation of models to identify an appropriate model form. 
General regression neural networks (GRNN), a second type of neural network, do not require the optimization of multiple parameters that is required in BPNN $(6,22)$. The GRNN models estimate appropriate model coefficients with a single pass of the data used in model development. GRNN models assign a nonparametric probability density function, in this case a Gaussian kernel, with width $s$, for each sample of independent and dependent variables. An appropriate value for $s$ is determined empirically based on mean square error between observed and estimated dependent variables (22). A joint probability estimate is converted to a conditional mean of dependent variables given the sample of independent variables, and the predicted value of dependent variables for future independent variables is assigned the most probable value given data used to develop the model $(6,22)$.

Neural networks have been applied to a wide range of prediction, optimization, and classification problems in agricultural sciences (18). In plant pathology, neural networks have been applied to the prediction of disease intensity $(1,7)$ and leaf wetness duration $(5,6,10)$.

Our objectives were to compare the disease-forecasting performance of BPNN, GRNN, logistic regression, nonparametric discriminant analysis, and two forms of parametric discriminant analysis, using tan spot and Stagonospora blotch as model systems. We have previously published a report of the potential application of neural network technology to disease forecasting and detailed how a BPNN could effectively model tan spot infection periods with an estimated accuracy level of $87 \%$ (7). Since this report, we have collected three additional years of disease-incidence data and refined our estimate of model accuracy, and we demonstrate here the potential of neural networks for disease forecasting.

\section{MATERIALS AND METHODS}

Infection-period bioassays were conducted during June and July of the 1993 through 1998 growing seasons at the North Dakota Agriculture Experiment Station in Fargo. Each year, a hard red spring wheat line, ND495, that is susceptible to both diseases was planted in clay pots $(20 \mathrm{~cm}$ diameter) in a greenhouse. Plants were fertilized with a time-release complete fertilizer and maintained in a greenhouse. When the main tillers of the plants reached the early milk growth stage (24), two pots were transported to a 1.0-ha wheat field planted with cv. Grandin (1993 to 1997) or cv. Kulm (1998). Both cultivars are considered susceptible to tan spot and Stagonospora blotch. Each year, wheat was planted into the debris of the previous year's wheat crop to promote disease development. The two pots were placed in submerged clay pots within the wheat crop, $\approx 10 \mathrm{~m}$ from field margins, to maintain a canopy microenvironment. After $24 \mathrm{~h}$ of exposure in the wheat field, plants were returned to the greenhouse, paired with two pots that had remained in the greenhouse, and placed in a growth chamber. The growth chamber was maintained at $21 \pm 2{ }^{\circ} \mathrm{C}$ with a 16 -h photoperiod $\left(400 \mu \mathrm{mol} \mathrm{s} \mathrm{s}^{-1} \mathrm{~m}^{-2}\right)$. Within the growth chamber, one of the fieldexposed pots and one of the pots that had remained in the greenhouse were exposed to an additional $24 \mathrm{~h}$ of wetness. All plants were watered by placing them in pans of water following their treatments, so the dry condition of the leaves could be maintained. After 10 days of incubation in the growth chamber, the top three leaves of two arbitrarily selected main tillers from each pot were observed for lesions. Leaves with lesions were surface-sterilized with a $1.0 \% \mathrm{NaOCl}$ solution for $3 \mathrm{~min}$, placed on $1.5 \%$ water agar, and incubated for approximately 14 days at $21^{\circ} \mathrm{C}$ with a $12-\mathrm{h}$ photoperiod. The identities of Pyrenophora tritici-repentis, Stagonospora nodorum, and Stagonospora avenaria were confirmed based on fruiting body and spore morphology. Disease incidence data for this modeling effort came from the field-exposed plants that did not receive any additional wetness. Further information regarding the infection period bioassay can be found in Francl (8) and De Wolf and Francl (7).
Environmental data for this study were obtained from the North Dakota Agricultural Weather Network (NDAWN). The NDAWN station was located $0.5 \mathrm{~km}$ from the wheat field and equipped with an automatic data logger with temperature, relative humidity, solar radiation, precipitation, and wind monitoring instrumentation (Campbell Scientific, Logan, UT). Information regarding leaf wetness was obtained from a second weather station located in the wheat field. This station was equipped with instrumentation nearly identical to that of the NDAWN station, except that replicate flat-plate resistance-type wetness sensors were added. All wetness sensors were painted off-white, positioned at flag-leaf height, and faced south at a $45^{\circ}$ angle. Any potential errors in the NDAWN data identified during quality control procedures were supplemented with information from the on-site weather station.

The data set consisted of 202 cases of the dependent variable tan spot or Stagonospora blotch incidence coded in a binary fashion (infection $=1$, no infection $=0$ ) and five associated independent variables derived from environmental data. Environmental variables were 24-h average temperature (degrees Celsius), and relative humidity (percent), 24-h sum of precipitation (millimeters), leaf wetness duration (hours), and accumulated growing degree days (2) from the time of planting. Leaf wetness duration was calculated by taking the $\log _{10}(x)$ of flat-plate sensor resistance (kiloohms) and applying a predetermined resistance threshold value of 2.95 to determine whether sensors were wet $(5,10)$. Hours of wetness were then summed across the 24-h period of plant exposure for each case.

The distribution and scale of independent variables were examined for undesirable skewness. Four of the five variables appeared to have acceptable distributions. However, the intermittent nature and magnitude of the precipitation variable was highly skewed. As a remedy, the precipitation values were transformed by taking the cube root. Each variable was rescaled between 1 and -1 , so all independent variables would be on the same scale. Finally, the independent variables were paired with the corresponding disease incidence data, and all variables were examined for linear correlation.

To develop the neural network and statistical models, the set of 202 cases were randomly assigned to a 152-case data set for model development or to a 50-case data set to validate model performance. The total number of cases was considered relatively low; therefore, potential influence of case randomization was avoided by repeating the randomization procedure nine additional times. This method of error estimation is similar to bootstrap error estimation procedures (20) but allows for evaluation and comparison of modeling methods with the same 10 random samples of available data.

Data were modeled with logistic regression, parametric, and nonparametric discriminant analysis (SAS Institute), backpropagation neural networks, and general regression neural networks. Logistic regression fits a symmetrical sigmoid function to a binary response with a maximum likelihood estimation to identify parameter estimates that most closely agree with observed data (13). Linear and quadratic discriminant analysis, two parametric methods, determine class membership by seeking either a linear or quadratic combination of variables that maximizes the ratio of between-group variance and within-group covariance (20). K-nearest neighbor $(\mathrm{KNN})$, a nonparametric method of discriminant analysis, makes no assumptions regarding data distributions. This method estimates class membership based on a proportion of the classes within a flexible number of small groups of observed cases presented during model development. The number of cases in each group is determined by a smoothing parameter, $k$. An appropriate value of $k$ was determined for each of the 10 data sets (SAS Institute). GRNN models were developed using software developed at North Dakota State University, Fargo (6). BPNN models were developed using NeuralWare Professional II Plus software (NeuralWare, Inc., Pittsburgh). Neural network models were implemented on a PC. 
Construction of the BPNN models involved evaluation of variable numbers of processing elements, transfer functions, and two model parameters, known as learning and momentum rates, that can potentially influence model performance. Initialization of model coefficients with a random number and presentation order of training cases can also affect model performance. To compensate for this potential variation, 10 replicate BPNN models were created with 1 to 10,20 , and 40 intermediate processing elements. The iterative processes involved in BPNN development can result in model predictions that are highly specific to data used during model development (3). A possible consequence of this data specificity is poor model validation performance. Thus, to avoid overspecification, models were periodically evaluated during the iterative network development by testing model performance on 38 of the 152 cases used in model development. The primary criterion for selection of the best BPNN models for each of the 10 data sets was validation prediction accuracy. Model performance also was evaluated using the secondary criteria of prediction accuracy of model development data and number of processing elements. All of the aforementioned statistical methods and neural networks were evaluated for prediction accuracy (number of correctly classified cases divided by total number cases) based on the midpoint between zero and one (i.e., 0.5). Mean validation prediction accuracies for modeling methods were evaluated by one-way analysis of variance (ANOVA) and least significant difference (LSD) mean separation identified groupings of treatment means where appropriate. Errors of the individual models with the best performance were observed for trends and correspondence with other information obtained from the infection period bioassay.

Average prediction accuracy of 10 replicate BPNN models with a single independent variable and two intermediate processing elements evaluated the importance of individual independent variables to model performance. In addition, prediction accuracy of 10 models with two intermediate processing elements evaluated the potential compensation of other variables by dropping the independent variable that resulted in the highest validation prediction accuracy. Only
1 of 10 data randomizations was used in this portion of the analysis, so comparisons could be made with the best BPNN models created with all independent variables.

\section{RESULTS}

In 202 potential infection periods, 103 of the plants exposed to the wheat-field environment had tan spot lesions, and 56 of the plants had Stagonospora blotch lesions (Table 1). Incidence of tan spot and Stagonospora blotch on field-exposed plants that received an additional $24 \mathrm{~h}$ of wetness was higher than incidence on plants exposed to the field alone. Correspondence between field-exposed plants and field-exposed plants that received an additional $24 \mathrm{~h}$ of wetness was 100 and $98 \%$ for tan spot and Stagonospora blotch, respectively. Two plants that remained in the greenhouse were infected with tan spot in 1994, and one plant was infected with Stagonospora blotch per year in 1993, 1994, and 1995. Plants that remained in the greenhouse but received an additional $24 \mathrm{~h}$ of wetness had infection levels intermediate to that of plants exposed to the wheat field and those that remained in the greenhouse but received no added wetness. The two occurrences of tan spot occurred on consecutive days and represent two and four lesions for these plants, whereas plants exposed to the field on the same days had 42 and 17 total lesions, respectively. The greenhouse plants with incidence of Stagonospora blotch had only a single lesion each in 1993 and 1995; however, infection occurred on only plants exposed to the wheat field and that received additional wetness. Plants that received other treatments were free of infection by Stagonospora blotch. The 1994 incidence of Stagonospora blotch corresponded with greenhouse tan spot incidence in the same year. This control plant had 12 Stagonospora lesions, in contrast to the 96 that occurred on the field-exposed plant. Incidence of Stagonospora blotch was more frequent during the early months of the growing season over the 6 years of the bioassay. In contrast, incidence of tan spot became more frequent toward the end of the growing season, particularly in 1996, 1997, and 1998.

TABLE 1. Percent incidence of tan spot and Stagonospora blotch on potted susceptible wheat plants exposed in a 1.0-ha wheat field for $24 \mathrm{~h}$ in Fargo, ND, during the 1993 through 1998 growing seasons

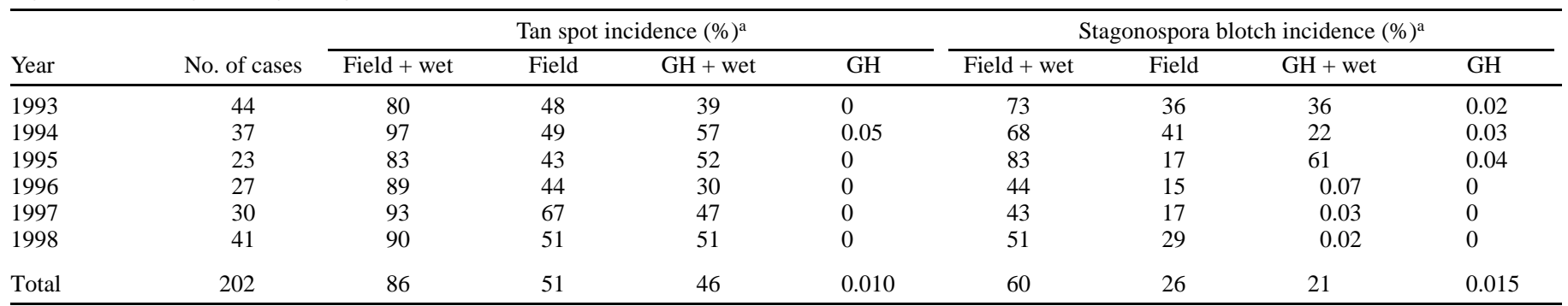

${ }^{a}$ Percent cases in which infection occurred on field-exposed wheat plants that were exposed to an additional $24 \mathrm{~h}$ of wetness in a growth chamber (field + wet); in which infection occurred on field-exposed wheat plants (field); in which infection occurred on plants that remained in the greenhouse and were exposed to an additional $24 \mathrm{~h}$ of wetness in a growth chamber ( $\mathrm{GH}+$ wet); and in which infection occurred on plants that remained in the greenhouse (GH).

TABLE 2. Mean prediction accuracy and standard error (SE) for statistical and neural network models of tan spot and Stagonospora blotch infection periods

\begin{tabular}{|c|c|c|c|c|c|c|c|c|}
\hline \multirow[b]{3}{*}{ Modeling approach } & \multicolumn{4}{|c|}{ Tan spot ${ }^{\mathrm{a}}$} & \multicolumn{4}{|c|}{ Stagonospora blotch ${ }^{\mathrm{a}}$} \\
\hline & \multicolumn{2}{|c|}{ Development } & \multicolumn{2}{|c|}{ Validation } & \multicolumn{2}{|c|}{ Development } & \multicolumn{2}{|c|}{ Validation } \\
\hline & Mean $(\%)$ & SE & Mean $(\%)$ & SE & Mean $(\%)$ & $\mathrm{SE}$ & Mean (\%) & SE \\
\hline Quadratic discriminant analysis & 78 & 0.42 & 72 & 1.85 & 78 & 0.57 & 76 & 2.74 \\
\hline Logistic regression & 77 & 0.43 & 71 & 2.11 & 83 & 0.63 & 81 & 1.77 \\
\hline General regression neural network & 78 & 0.81 & 72 & 1.86 & 78 & 0.82 & 78 & 2.23 \\
\hline K-nearest neighbor & 83 & 1.40 & 74 & 1.12 & 81 & 1.46 & 76 & 2.50 \\
\hline Protected LSD at $P=0.01^{\mathrm{b}}$ & $\ldots$ & $\ldots$ & NS & $\ldots$ & $\ldots$ & $\ldots$ & 6.3 & $\cdots$ \\
\hline
\end{tabular}

a Data from the 1993 through 1998 growing seasons were randomly assigned to data sets used in either model development $(n=152)$ or validation $(n=50)$.

Mean prediction accuracy and SE were based on the prediction accuracy of models developed and validated with 10 randomizations of 202 cases.

b LSD = least significant difference; $\ldots=$ statistic was not calculated; NS = not significant. 
The highest linear correlation between independent and dependent variables was 0.49 for both hours of leaf wetness duration and tan spot incidence and precipitation and Stagonospora blotch. The highest correlation between two independent variables was 0.43 for relative humidity and precipitation; all other correlations were $<0.36$. Correlation coefficients $\geq 0.18$ were significant at $P=0.01$.

Optimization of the BPNN models by varying the number the processing elements between 1 to 10,20 , or 40 resulted in only small changes (often less than 1\%) in validation prediction accuracy for either disease. No positive or negative trend could be detected based on evaluation of BPNN models with other transfer functions, learning rates, or momentum rates. Therefore, the criteria of prediction accuracy for validation and development data sets
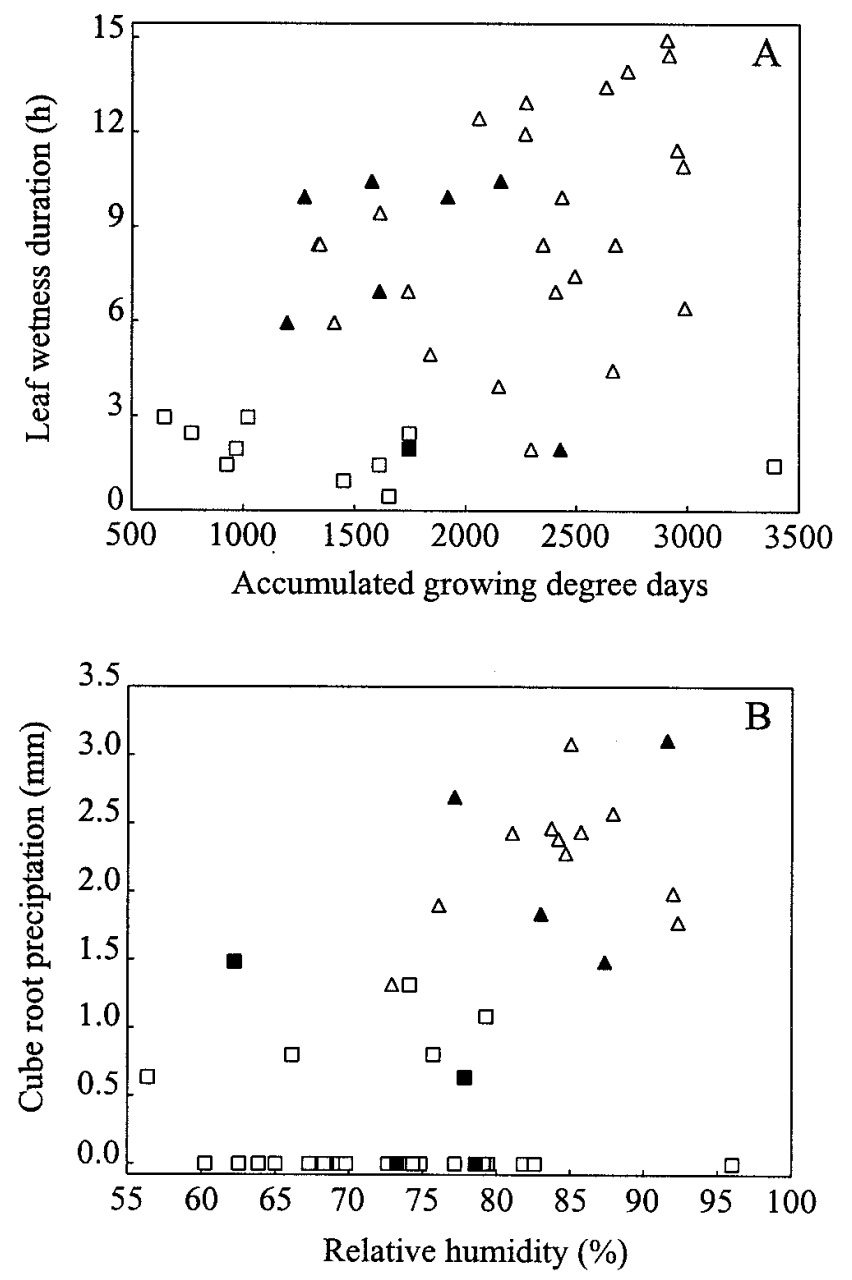

Fig. 1. Backpropagation neural network performance on validation data for correspondence between observed and predicted A, tan spot and B, Stagonospora blotch incidence on bioassay plants exposed to a wheat-field environment for $24 \mathrm{~h}$. Variables used to construct plot axes were identified as the most important to disease prediction based on sensitivity analyses. $\Delta=$ correctly predicted infection; $\boldsymbol{\Delta}=$ false positives; $\square=$ correctly predicted noninfection; and $\mathbf{\square}=$ false negatives. was deemed as more informative than the optimization of the number of processing elements.

Logistic regression, linear discriminant, quadratic discriminant, and GRNN models had a mean prediction accuracy within 1 and $2 \%$ for tan spot validation and development data sets (Table 2). The KNN models had a slightly higher mean prediction accuracy for both model validation and development data sets than the parametric statistical methods. In comparison, BPNN models had a mean prediction accuracy similar to those of the statistical models for data used in model development but had the highest mean validation prediction accuracy. Mean validation prediction accuracy for tan spot infection periods was not significantly different $(P=0.42)$ among models.

The best BPNN model of tan spot infection periods had seven processing elements in the processing layer and correctly classified $78 \%$ of the model development cases and $82 \%$ of the validation cases. This BPNN model had nine erroneous validation observations. Eight of the errors were false positives and one was a false negative (Fig. 1). Seven of the false positives had leaf wetness duration of $\geq 6 \mathrm{~h}$. Five of the false positives occurred early in the growing season. Another of the false positives had no infection on corresponding field-exposed plants that received an additional $24 \mathrm{~h}$ of wetness, suggesting that absence of inoculum may have been the limiting factor. Both the one remaining false positive and the false negative had $2 \mathrm{~h}$ of leaf wetness; however, they also had a average relative humidity of $\approx 80 \%$.

The mean validation prediction accuracy of Stagonospora blotch infection periods for linear discriminant, quadratic discriminant, and KNN models was within $1 \%$. Mean validation prediction accuracy for the GRNN models was slightly (2\%) higher relative to the discriminant methods, but mean prediction accuracy for developmental data sets was identical to parametric-discriminant and GRNN methods. BPNN models had the highest mean prediction validation accuracy, correctly classifying an average of $86 \%$ of the validation cases. Prediction accuracy for logistic regression models was intermediate to the BPNN and other modeling methods. Mean prediction accuracy of BPNN models for development cases was $1 \%$ less than the corresponding result for the logistic regression models. Mean validation prediction accuracy among models was significantly different $(P=0.01)$.

The best BPNN model of Stagonospora blotch had six processing elements in the processing layer and correctly classified 85 and $84 \%$ of the development and validation cases, respectively. This model incorrectly classified eight validation cases, with four false positives and four false negatives (Fig. 1). All false-positive errors were associated with $\geq 10 \mathrm{~h}$ of leaf wetness duration and $\geq 3.4 \mathrm{~mm}$ of precipitation. In comparison, false-negative predictions by the model were associated with $\leq 3.4 \mathrm{~mm}$ of precipitation, $\leq 9 \mathrm{~h}$ of leaf wetness duration, and $\leq 79 \%$ relative humidity. No temporal trend in model error distribution was observed.

Leaf wetness duration and accumulated growing degree days predicted validation cases of tan spot with $\geq 72 \%$ average accuracy in a sensitivity analysis of single independent variable BPNN models (Table 3). All other independent variables had average prediction accuracies of $\leq 58 \%$ for tan spot incidence. When the leaf wetness duration variable was withheld from the best BPNN model, pre-

TABLE 3. Sensitivity analysis of input variables to neural network models of tan spot and Stagonospora blotch infection periods

\begin{tabular}{|c|c|c|c|c|}
\hline \multirow[b]{2}{*}{ Variable } & \multicolumn{2}{|c|}{ Tan spot ${ }^{\mathrm{a}}$} & \multicolumn{2}{|c|}{ Stagonospora blotch ${ }^{\mathrm{a}}$} \\
\hline & Development (\%) & Validation (\%) & Development (\%) & Validation $(\%)$ \\
\hline Leaf wetness duration (h) & 73 & 77 & 75 & 68 \\
\hline Precipitation $(\mathrm{mm})$ & 71 & 51 & 77 & 80 \\
\hline Accumulated growing-degree days & 67 & 72 & 66 & 64 \\
\hline Relative humidity $(\%)$ & 59 & 58 & 77 & 75 \\
\hline Temperature $\left({ }^{\circ} \mathrm{C}\right)$ & 56 & 58 & 65 & 58 \\
\hline
\end{tabular}

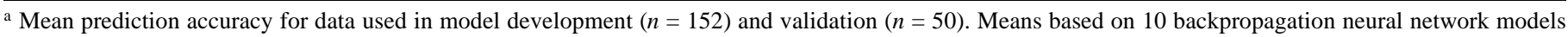
developed and validated with a single randomization of 202 potential infection periods. 
diction accuracy on validation cases decreased by $20 \%$ compared to the full model. For Stagonospora blotch, precipitation and relative humidity predicted validation cases with $\geq 75 \%$ average accuracy. Other variables had lower prediction accuracies. When BPNN models without the precipitation variable were evaluated, validation prediction accuracy decreased by $6 \%$ relative to the best BPNN model.

When 10 validation trials were averaged, BPNN models of tan spot incidence had a higher classification accuracy for cases with infection (84\%) than without infection (67\%). In contrast, BPNN models of Stagonospora blotch had a higher prediction accuracy for cases without infection $(86 \%)$ than with infection $(61 \%)$.

\section{DISCUSSION}

Close correspondence between the infection of field-exposed bioassay plants and exposed plants that received additional postexposure wetness provides evidence that tan spot and Stagonospora blotch lesions on the former treatment were the result of an environment favorable for inoculation and infection. Therefore, we conclude that the bioassay effectively monitored infection events in the wheat-field environment. The increased frequency of tan spot incidence on field-exposed plants late in a growing season may be the result of increasing inoculum levels often observed for tan spot during those times $(9,17)$. Similarly, the high Stagonospora blotch incidence rate during the early months of a growing season may be explained, in part, by frequent precipitation events in North Dakota during these time periods that favor the splash dispersal of Phaeosphaeria nodorum pycnidiospores (14).

In general, BPNN models resulted in superior classification of both tan spot and Stagonospora blotch infection periods based on the comparison of mean validation prediction accuracy. More specifically, all of the evaluated methods of modeling tan spot infection periods were not statistically different based on comparison of mean validation prediction accuracy. The $76 \%$ mean validation accuracy and $82 \%$ accuracy of the best BPNN model were less than the optimal accuracy previously estimated at $87 \%$ (7). However, BPNN models had the highest mean validation prediction accuracy among modeling methods. Based on this estimate of global model error, we conclude that the BPNN method is the best choice. Unlike the models for tan spot, comparison of mean validation prediction accuracy for methods of modeling Stagonospora blotch were significantly different. Mean separation procedures narrowed the selection of the best modeling method to either logistic regression or BPNN. BPNN models had the highest mean validation prediction accuracy; therefore, we again conclude that the BPNN method is the best choice. Although logistic regression and KNN methods were comparable for one of the diseases, neither performed well under either circumstance. The GRNN is a robust connectionist method, but the current data set may have been too sparse for optimal model performance.

Independent variables identified by sensitivity analysis correspond well with variables identified as being important during controlled experimentation for both tan spot and Stagonospora blotch. In the case of the tan spot model, the importance of leaf wetness duration to model accuracy and observation of model errors in relation to other bioassay data indicate that BPNN identified critical values of wetness duration similar to the $6 \mathrm{~h}$ threshold identified by Hosford et al. (12). The importance of precipitation and relative humidity to the accuracy of the Stagonospora blotch model corresponded well with precipitation and relative humidity thresholds reported by Jeger et al. (14). However, the unimportance of wetness to the Stagonospora blotch model and temperature to both models appears to conflict with these previous reports $(12,14)$. The response to temperature during the infection process may have gone undetected by BPNN because of the low number of discrete periods in which temperature was a potentially limiting factor. The unimportance of the wetness variable to the Stagono- spora blotch model may be explained, in part, by the prior requirement of splash dispersal and deposition of pycnidiospores onto field-exposed plants. On two occasions, Stagonospora blotch occurred in the absence of recorded precipitation, a result misclassified by the neural network (Fig. 1) and due perhaps to physical contact between healthy and diseased leaves in the field or during incubation. The greater number of false-positive model predictions made at accumulated growing degree-day values less than 2,000 may indicate that the tan spot model was unable to detect the upward seasonal trend in inoculum levels (9).

Although the accuracy of the BPNN models appears to be acceptable for application in disease forecasting, successful adaptation of an infection-period model into a forecasting system will require research to determine at what growth stage to start the model and how many infection periods can be allowed to accumulate prior to fungicide application. In addition, these infectionperiod thresholds should be evaluated for different management strategies, such as crop rotation and tillage practices, that reduce disease pressure. As with all empirical models, BPNN performance could decrease if information used to develop the model does not adequately represent potential variability in the system. Therefore, a continued evaluation of model performance over a broader range of environments and in other regions prior to its integration into a forecasting system is advisable. BPNN models produced by NeuralWare software can be implemented in "C code" programs that parameterize the models for new independent-variable data. The final programs then can be implemented on a networked computer, which should facilitate model application.

Wheat yield response to a fungicide involves appropriate application timing and anticipation of future infection probabilities. The profit margin for spring wheat and durum is often narrow (11); therefore, only the most accurate forecasting system possible would be acceptable to risk-averse producers. Neural networks may be part of this system in North Dakota because they consistently performed better than other modeling methods. In the future, neural networks may be applied advantageously in other forecasting systems.

\section{LITERATURE CITED}

1. Batchelor, W. D. Yang, X. B., and Tschanz, A. T. 1997. Development of a neural network for soybean rust epidemics. Trans. ASAE 40:247-252.

2. Bauer, A., Fanning, C. D., Enz, J. W., and Eberlin, C. V. 1984. Use of growing degree days to determine spring wheat growth stages. N.D. State Univ. Coop. Ext. Bull. 37.

3. Bishop, C. M. 1996. Neural Networks for Pattern Recognition. Oxford University Press, New York.

4. Bockus, W. W., Davis, M. A., and Shroyer, J. P. 1992. Effect of foliar fungicide application on seed size of winter wheat. J. Appl. Seed Prod. 10:1-6.

5. Chtioui, Y., Francl, L. J., and Panigrahi, S. 1999. Moisture prediction from simple micrometeorological data. Phytopathology 89:668-672.

6. Chtioui, Y., Panigrahi, S., and Francl, L. 1999. A generalized regression neural network and its application for leaf wetness prediction to forecast plant disease. Chemometrics Intel. Lab. Syst. 48:47-58.

7. De Wolf, E. D., and Francl, L. J. 1997. Neural networks that distinguish infection periods of wheat tan spot in an outdoor environment. Phytopathology 87:83-87.

8. Francl, L. J. 1995. Challenge of bioassay plants in a monitored outdoor environment. Can. J. Plant Pathol. 17:138-143.

9. Francl, L. J. 1997. Local and mesodistance dispersal of Pyrenophora tritici-repentis. Can. J. Plant Pathol. 19:247-255.

10. Francl, L. J., and Panigrahi, S. 1997. Artificial neural network models of wheat leaf wetness. Agric. For. Meteorol. 88:57-65.

11. Haugen, R. H., and Aakre, D. G. 1999. Cost-benefit and statistical analysis of fungicides for suppression of Fusarium head blight (scab) in hard red spring wheat in 1997. N.D. State Univ. Agric. Econ. Rep. 184.

12. Hosford, R. M., Jr., Larez, C. R., and Hammond, J. J. 1987. Interaction of wet period and temperature on Pyrenophora tritici-repentis infection and development in wheats of differing resistance. Phytopathology 77: 1021-1027.

13. Hosmer, D. W., Jr., and Lemeshow, S. 1989. Applied Logistic Regression. Wiley Interscience, New York.

14. Jeger, M. J. Griffiths, W., and Jones, D. G. 1981. Influence of environmental conditions on spore dispersal and infection by Septoria nodorum. 
Ann. Appl. Biol. 99:29-34.

15. Kohli, M. M., Mehta, Y. R., and de Ackermann, M. D. 1992. Spread of tan spot in the southern cone region of South America. Pages. 86-90 in: Advances in Tan Spot Research. L. J. Francl, J. M. Krupinsky, and M. P. McMullen, eds. North Dakota State University Agricultural Experiment Station, Fargo.

16. McMullen, M. P., and Nelson, D. R. 1992. Tan spot and five years of wheat disease survey. Pages 80-85 in: Advances in Tan Spot Research. L. J. Francl, J. M. Krupinsky, and M. P. McMullen, eds. North Dakota State University Agricultural Experiment Station, Fargo.

17. Morrall, R. A. A., and Howard, R. J. 1975. The epidemiology of leaf spot disease in a native prarie. II. Airborne spore populations of Pyrenophora tritici-repentis. Can. J. Bot. 53:2345-2353.

18. Panigrahi, S. 1998. Neuro-fuzzy systems: Applications and potential in biology and agriculture. Artif. Intelligence Appl. 12:83-95.

19. Rees, R. G., and Platz G. J. 1980. The epidemiology of yellow spot of wheat in southern Queensland. Aust. J. Agric. Res. 31:259-267.

20. Ripley, B. D. 1996. Pattern Recognition and Neural Networks. Cambridge University Press, New York.

21. Shipton, W. A., Boyd, W. R. J., Rosielle, A. A., and Shearer, B. L. 1971. The common Septoria diseases of wheat. Bot. Rev. 37:231-262.

22. Specht, D. F. 1991. A general regression neural network. IEEE Trans. Neural Networks 2:568-576.

23. Stover, R. W., Francl, L. J., and Jordahl, J. G. 1996. Tillage and fungicide management of foliar diseases in a spring wheat monoculture. J. Prod. Agric. 9:261-265.

24. Zadoks, J. C., Chang, T. T., and Konzak, C. F. 1974. A decimal code for the growth stage of cereals. Weed Res. 14:415-421. 